\title{
Childhood functional gastrointestinal disorders
}

\author{
A Rasquin-Weber, P E Hyman, S Cucchiara, D R Fleisher, J S Hyams, P J Milla, A Staiano
}

Co-Chair, Committee on Childhood

Functional

Gastrointestinal

Disorders,

Multinational Working

Teams to Develop

Criteria for Functional

Disorders (Rome II),

Professor of

Pediatrics,

University of

Montreal,

Montreal, Canada

A Rasquin-Weber

Chair, Committee on Childhood Functional Gastrointestinal

Disorders,

Multinational Working

Teams to Develop

Criteria for Functional

Disorders (Rome II),

Associate Clinical

Professor of

Pediatrics,

University of

California at Los

Angeles,

Orange County, CA,

USA

P E Hyman

Associate Professor of Pediatrics,

University Frederico II, Naples, Italy

S Cucchiara

Associate Professor of Child Health,

University of Missouri,

Columbia, MO, USA

D R Fleisher

Professor and Vice Chair of Pediatrics, University of

Connecticut School of

Medicine,

Hartford, CT, USA

J S Hyams

Reader in Pediatric

Gastroenterology,

University of London,

London, UK

P J Milla

Associate Professor of Pediatrics,

University Frederico

II, School of Medicine,

Naples, Italy

A Staiano

Correspondence to:

Andree Rasquin-Weber, MD, Gastrointestinal Division,

Hopital Ste Justine, 3175 St

Catherine Road, Montreal,

Quebec H3T 1C5, Canada.
Abstract

This is the first attempt at defining criteria for functional gastrointestinal disorders (FGIDs) in infancy, childhood, and adolescence. The decision-making process was as for adults and consisted of arriving at consensus, based on clinical experience. This paper is intended to be a quick reference. The classification system selected differs from the one used in the adult population in that it is organized according to main complaints instead of being organ-targeted. Because the child is still developing, some disorders such as toddler's diarrhea (or functional diarrhea) are linked to certain physiologic stages; others may result from behavioral responses to sphincter function acquisition such as fecal retention; others will only be recognizable after the child is cognitively mature enough to report the symptoms (e.g., dyspepsia). Infant regurgitation, rumination, and cyclic vomiting constitute the vomiting disorders. Abdominal pain disorders are classified as: functional dyspepsia, irritable bowel syndrome (IBS), functional abdominal pain, abdominal migraine, and aerophagia. Disorders of defecation include: infant dyschezia, functional constipation, functional fecal retention, and functional nonretentive fecal soiling. Some disorders, such as IBS and dyspepsia and functional abdominal pain, are exact replications of the adult criteria because there are enough data to confirm that they represent specific and similar disorders in pediatrics. Other disorders not included in the pediatric classification, such as functional biliary disorders, do occur in children; however, existing data are insufficient to warrant including them at the present time. For these disorders, it is suggested that, for the time being, clinicians refer to the criteria established for the adult population.

(Gut 1999;45(Suppl II):II60-II68)

Keywords: infant vomiting; cyclic vomiting syndrome; functional dyspepsia in children; irritable bowel syndrome in children; functional abdominal pain in children; functional diarrhea in children; functional constipation in children; Rome II

The pediatric working team met for the first time in Rome in September 1997, seven years after the first paper classifying diagnostic criteria for functional gastrointestinal disorders (FGIDs) in the adult population was published. ${ }^{1}$ These disorders were further defined in a document, now referred to as the Rome criteria for FGIDs. ${ }^{2}$ These criteria have provided clinicians with a method for standardizing their manner of defining clinical disorders, and have allowed researchers from various fields to study the physiology and treatment of the same disorders from different points of view. Publication of the Rome diagnostic criteria resulted in an explosion of clinical research, contributed to an improved understanding of FGIDs, and provided clinicians with a positive approach to treating patients. $^{3}$

It was perceived as a privilege by our working team to be offered the challenge of defining diagnostic criteria according to the Rome criteria for the pediatric population. We believed that a collaboration with our adult gastroenterology colleagues would increase our understanding of FGIDs and provide a basis for longitudinal studies on the origins and evolution of these disorders.

Childhood FGIDs include a variable combination of often age-dependent, chronic, or recurrent symptoms not explained by structural or biochemical abnormalities. As the child is programmed to develop, it is not surprising that some functional disorders which occur during childhood accompany normal development (e.g., infant regurgitation or toddler's diarrhea), or may be triggered by age appropriate but maladaptive behavioral responses to internal or external stimuli (e.g., functional fecal retention often results from painful defecation and/or coercive toilet training). The diagnosis of some childhood FGIDs depends on the child's ability to report symptoms. Thus some disorders, such as irritable bowel syndrome (IBS), are not described in children below a certain age. This does not preclude its existence in younger children; rather, preschool children are unable to report the necessary details for a diagnosis. Therefore, instead of classifying disorders according to target organs (as in the adult population), we divided pediatric disorders according to main complaints reported by children or their parents. For example, aerophagia was classified within pediatric disorders as presenting as abdominal pain, whereas in adults, the disorder was classified as belonging to the esophagus-related group of symptoms.

The working team agreed that some infants inherit a temperament characterized in part by gastrointestinal reactivity to stress, which constitutes a genetic susceptibility to FGIDs. Indeed, temperament-sensitive reactivity in infants has already been suggested in association with three other biological systems (cardiovascular, neuroendocrine, and immunologic). ${ }^{4}$ Conversely, our committee

Abbreviations used in this paper: FGID, functional gastrointestinal disorder; IBS, irritable bowel syndrome; RAP, recurrent abdominal pain. 
Table 1 Functional gastrointestinal disorders

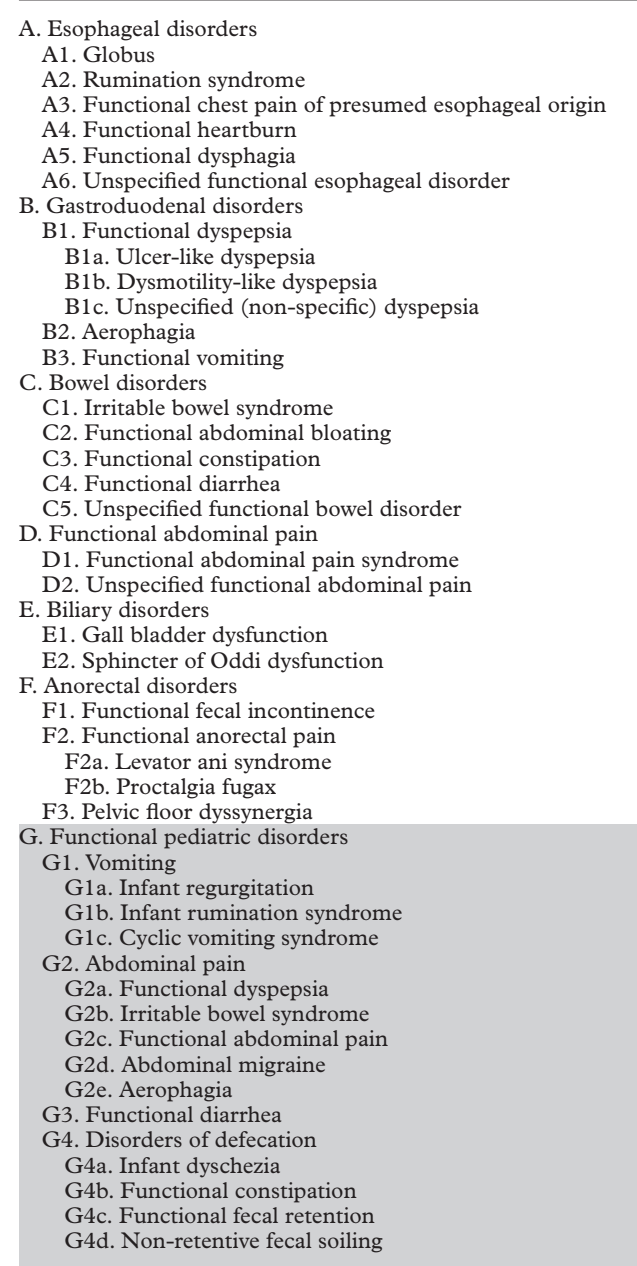

members were convinced that environmental factors during early life also play a role in the development of FGIDs. Firstly, plasticity of the neonatal brain allows early life events to program physiologic responses to stress during infancy, and these responses may be perpetuated into adulthood. ${ }^{5}$ Secondly, children learn illness-related attitudes and behaviors from their parents and caretakers. Health care utilization by children closely resembles that of their parents. ${ }^{6}$ Thus, not only should treatment for children include their parents, but the family should be taught about the role that psychosocial factors play in the development and perpetuation of FGIDs.

This paper summarizes the diagnostic criteria for pediatric FGIDs, and is intended to be a quick reference (table 1). Therefore, only clinical, diagnostic, and treatment recommendations are included. The reader is referred to other publications for detailed clinical, epidemiologic, physiologic, and psychological considerations. ${ }^{78}$ For certain disorders (e.g., IBS and functional dyspepsia), criteria in the adult population were replicated exactly for the pediatric population because they seemed to apply equally well to children. FGIDs other than those mentioned in this article occur during childhood, but were defined adequately by the adult criteria. These include globus, functional chest pain, functional heartburn, functional dysphagia, and proctalgia fugax. Functional biliary disorders were not described because documentation and experience with gall bladder and sphincter of Oddi dysfunction in children are insufficient. Eventually, it was agreed that the co-occurrence of FGIDs and organic disease in the same child often goes unrecognized. Thus, for example, the presence of IBS often leads to overtreatment in adolescents with inflammatory bowel disease.

\section{G1. Vomiting}

\section{G1a. Infant regurgitation}

Regurgitation is the involuntary return of previously swallowed food or secretions into or out of the mouth. Regurgitation is distinguished from vomiting, which is defined by a central nervous system reflex involving both autonomic and skeletal muscles in which gastric contents are forcefully expelled through the mouth because of coordinated movements of the small bowel, stomach, esophagus, and diaphragm. Regurgitation, vomiting, and rumination are examples of gastroesophageal reflux. When the latter causes or contributes to tissue damage or inflammation (e.g. esophagitis, obstructive apnea, reactive airway disease, pulmonary aspiration, or failure to thrive), it is called gastroesophageal reflux disease.

DIAGNOSTIC CRITERIA

(1) Regurgitation two or more times per day for three or more weeks;

(2) There is no retching, hematemesis, aspiration, apnea, failure to thrive, or abnormal posturing;

(3) The infant must be $1-12$ months of age and otherwise healthy;

(4) There is no evidence of metabolic, gastrointestinal, or central nervous system disease to explain the symptom.

CLINICAL AND DIAGNOSTIC RECOMMENDATIONS Prematurity, developmental delay, and congenital abnormalities of the oropharynx, chest, lungs, central nervous system, or gastrointestinal tract are considered risk factors for gastroesophageal reflux disease. Accompanying systemic conditions (milk allergy) may be suspected when eczema is present; an abnormal neurological examination should prompt further investigation. A diagnosis of gastroesophageal reflux disease should be evoked in the presence of failure to thrive, hematemesis, occult blood in the stool, anemia, or refusal to eat. However, chronic regurgitation, early satiety, refusal of food, and excessive crying, sometimes leading to failure to thrive, may be the physical consequences of emotional distress. ${ }^{9}$

\section{TREATMENT}

Since infant regurgitation is a transient problem, possibly due in part to the immaturity of gastrointestinal motility, treatment goals are to provide effective reassurance and symptom relief. Symptoms often improve with prone 
positioning after meals, ${ }^{10}$ formula thickened with cereal, ${ }^{11}$ smaller volume feedings, and drugs that improve motility such as cisapride. ${ }^{12}$

\section{G1b. Infant rumination syndrome}

Rumination is a rare disorder characterized by the voluntary, habitual regurgitation of stomach contents into the mouth for self-stimulation. ${ }^{13}$

DIAGNOSTIC CRITERIA

(1) Infant rumination syndrome is defined by at least three months of stereotypical behavior beginning with repetitive contractions of the abdominal muscles, diaphragm, and tongue, and culminating in regurgitation of gastric contents into the mouth which is either expectorated or rechewed and reswallowed, and three or more of the following:

(a) Onset between three and eight months of age;

(b) Does not respond to management for gastroesophageal reflux disease, anticholinergic drugs, hand restraints, formula changes, and gavage or gastrostomy feedings;

(c) Unaccompanied by signs of nausea or distress; and/or

(d) Does not occur during sleep and when the infant is interacting with individuals in the environment.

CLINICAL AND DIAGNOSTIC RECOMMENDATIONS Observation of the ruminative act is all that is needed to make the diagnosis. This might be difficult as rumination may cease as soon as the infant notices the observer. Parents will not provide the information spontaneously, but when asked about the possibility of stereotypical behavior, they will recognize it. The infant who ruminates may not retain enough nutrients and may develop potentially lethal malnutrition, a complication that seldom, if ever, occurs in older ruminators. ${ }^{13}$ Sensory and/or emotional deprivation characterize the child with rumination. This explains why the disorder occurs in institutionalized children and infants in intensive care units, as well as in normal infants from emotionally distant mothers.

TREATMENT

Treatment efforts should be directed toward caregivers as well as the child. In infant rumination, the relationship between the infant and caregiver is devoid of enjoyment. The goal of therapy is to provide a nurturing environment and comforting care to the infant, and to help the mother change her feelings towards herself and her infant. This can be achieved by improving her ability to recognize and respond sensitively to her infant's physical and emotional needs. In mentally handicapped children, providing a nurturing caregiver may not be sufficient; behavioral therapy involving positive reinforcement and possibly aversive techniques may be necessary. ${ }^{14}$

\section{G1c. Cyclic vomiting syndrome}

Cyclic vomiting syndrome consists of recurrent, stereotypical episodes of intense nausea and vomiting lasting hours to days, which are separated by symptom-free intervals. The frequency of episodes averages 12 per year (range 1-70) and intervals between attacks may be fairly regular or sporadic. Episodes tend to begin at the same time of day, usually during the night or early morning. The duration of episodes tends to be the same in each patient over months or years. ${ }^{15}$ Once vomiting begins, it reaches its highest intensity during the first hours. ${ }^{16}$

DIAGNOSTIC CRITERIA

(1) A history of three or more periods of intense, acute nausea, and unremitting vomiting lasting hours to days, with intervening symptom-free intervals, lasting weeks to months.

(2) There is no metabolic, gastrointestinal or central nervous system structural or biochemical disease.

CLINICAL AND DIAGNOSTIC RECOMMENDATIONS Typically, cyclic vomiting occurs in a child aged 2-7 years (with a possible range from infancy to mid-life), who is free of vomiting between episodes. However, like their family members, these children often complain of migraines, motion sickness, and other functional bowel disorders. During vomiting episodes, accompanying signs and symptoms include pallor, weakness, increased salivation, abdominal pain, intolerance to noise, light or odors, headache, diarrhea, fever, tachychardia, hypertension, skin blotching, and leukocytosis. A triggering factor (emotions, infection) can be identified in $80 \%$ of cases. Differential diagnosis should include brain stem tumors, obstructive uropathy, peptic disease, recurrent pancreatitis, intermittent bowel obstruction, chronic intestinal pseudo-obstruction, and familial dysautonomia. Among metabolic and endocrine diseases, those which may mimic cyclic vomiting include pheochromocytoma, adrenal insufficiency, diabetes mellitus, urea cycle enzyme deficiency, medium-chain acyl coenzyme A dehydrogenase deficiency, proprionic acidemia, and porphyria.

\section{TREATMENT}

Emotional conditions which trigger episodes should be identified and treated. When episodes are frequent and severe, prophylactic daily treatment with cyproheptadine, amitriptyline, erythromycin, phenobarbital, sumatripan, or propanolol may succeed in reducing frequency or eliminating episodes. ${ }^{17-19}$ In some children with a recognizable prodrome, oral medications such as ondansetron, erythromycin, and ibuprofen may be useful prior to onset of nausea. In patients whose episodes cannot be prevented, it may be helpful to begin, as early as possible, an oral acid inhibiting drug to protect esophageal mucosa and dental enamel, and lorazepam for its anxiolytic, sedative, and anti-emetic effects. Intravenous 
ondansetron, granisetron, diphenhydramine, and chlorpromazine may also be helpful and will sometimes interrupt the crisis. The presence of electrolyte deficits, hypertension, and/or inappropriate secretion of antidiuretic hormone should be detected and treated.

\section{G2. Abdominal pain}

The pediatric working team achieved consensus and chose not to include infant colic ${ }^{20}$ and recurrent abdominal pain (RAP) of childhood ${ }^{21}$ as FGIDs. In the case of colic, there is no evidence that the gastrointestinal tract is involved..$^{20}$ In the case of RAP, three reasons were invoked to exclude it: (1) Appley's criteria are too general; (2) symptoms in school-aged children with chronic or recurrent abdominal pain often meet the Rome criteria for functional dyspepsia, IBS, or functional abdominal pain in adults. ${ }^{22}$ It seemed appropriate to apply the most specific diagnostic category to a symptomatic child, so that we may learn more about the natural history of these FGIDs with childhood onset, and consider novel intervention strategies for children who meet diagnostic criteria for these conditions; and (3) there is a growing body of evidence to suggest that functional abdominal pain is often associated with visceral hyperalgesia, a reduced threshold for pain related to biochemical changes in the afferent neurons of the enteric and central nervous systems. ${ }^{23}$ Despite the fact that evidence for visceral hyperalgesia in children with functional abdominal pain remains preliminary, we are currently using this theory to explain the pathogenesis of pain, in order to alleviate parents' feelings of guilt and concern that the problem is psychological.

\section{G2a. Functional dyspepsia}

Dyspepsia refers to pain or discomfort centered in the upper abdomen (see also functional dyspepsia in adults). Discomfort may be characterized by fullness, early satiety, bloating, belching, queasiness, nausea, retching, or vomiting. These symptoms typically include a component of upper abdominal distress. ${ }^{24}$

DIAGNOSTIC CRITERIA

Functional dyspepsia has not been rigorously defined in a pediatric population. Therefore we have adopted the adult diagnostic criteria for use in children.

In children mature enough to provide an accurate pain history, at least 12 weeks, which need not be consecutive, within the preceding 12 months of:

(1) Persistent or recurrent pain or discomfort centered in the upper abdomen (above the umbilicus); and

(2) No evidence (including at upper endoscopy) that organic disease is likely to explain the symptoms; and

(3) No evidence that dyspepsia is exclusively relieved by defecation or associated with the onset of a change in stool frequency or stool form.
The concept of subdividing functional dyspepsia based on distinctive features may be useful. These divisions seem especially relevant to those proposing to study this FGID.

\section{G2a1. Ulcer-like dyspepsia}

Pain centered in the upper abdomen is the predominant (most bothersome) symptom.

\section{G2a2. Dysmotility-like dyspepsia}

An unpleasant or troublesome non-painful sensation (discomfort) centered in the upper abdomen is the predominant symptom; this sensation may be characterized by or associated with upper abdominal fullness, early satiety, bloating, or nausea.

\section{G2a3. Unspecified (non-specific)} dyspepsia

Symptomatic patients whose symptoms do not fulfil the criteria for either ulcer-like or dysmotility-like dyspepsia.

CLINICAL AND DIAGNOSTIC RECOMMENDATIONS In children mature enough to provide an accurate pain history, the clinician conducts an interview which includes queries about dietary, psychological, and social factors. The clinician determines whether symptoms are likely to represent mucosal disease (esophagitis, gastritis, duodenitis, ulcer). Demographic and familial data should raise suspicion of Helicobacter pylori infection. ${ }^{25}$ Endoscopy will confirm or eliminate these diagnoses. A previous episode of viral infection may suggest post-viral gastroparesis. ${ }^{26}$

Measuring serum amylase, lipase, and aminotransferase concentrations, as well as performing an abdominal ultrasound, aid in assessing the presence of pancreatic, liver, or biliary disease. In functional dyspepsia, physical examination and growth are normal and there are no signs of inflammatory bowel disease.

\section{TREATMENT}

There are no controlled treatment trials for functional dyspepsia in children. Medication and food known to aggravate symptoms should be discontinued. Histamine receptor antagonists, proton pump inhibitors, sucralfate, and low dose tricyclic antidepressants have been used. Prokinetic drugs such as cisapride may be helpful for feelings of fullness and metoclopramide may alleviate nausea. If psychological stress aggravates symptoms, the clinician and family should collaborate on a plan to reduce the stress.

\section{G2b. Irritable bowel syndrome}

In IBS (see also criteria for irritable bowel syndrome in adults) abdominal discomfort or pain is associated with defecation or a change 
in bowel habit, with features of disordered defecation. Ten to $20 \%$ of adolescentsand adults have symptoms consistent with IBS. ${ }^{22}$

\section{DIAGNOSTIC CRITERIA}

In children old enough to provide an accurate pain history, at least 12 weeks, which need not be consecutive, in the preceding 12 months of;

(1) Abdominal discomfort or pain that has two out of three features:

(a) Relieved with defecation; and/or

(b) Onset associated with a change in frequency of stool; and/or

(c) Onset associated with a change in form (appearance) of stool; and

(2) There are no structural or metabolic abnormalities to explain the symptoms.

The following symptoms cumulatively support the diagnosis of IBS:

- Abnormal stool frequency (for research purposes "abnormal" may be defined as greater than three bowel movements per day and less than three bowel movements per week);

- abnormal stool form (lumpy/hard or loose/ watery stool);

- abnormal stool passage (straining, urgency, or feeling of incomplete evacuation);

- passage of mucus;

- bloating or feeling of abdominal distension.

CLINICAL AND DIAGNOSTIC RECOMMENDATIONS

A history which fits the Rome criteria for a diagnosis of IBS, accompanied by a normal physical examination and growth, is consistent with a diagnosis of childhood IBS. A nutritional history, assessing for adequacy of dietary fiber in those with constipation, as well as ingestion of sugars such as sorbitol and fructose in those with diarrhea, is often useful. Factors alerting the clinician to the possibility of disease include nocturnal pain or diarrhea, weight loss, rectal bleeding, fever, arthritis, delayed puberty, and a family history of inflammatory bowel disease. Limited laboratory screening for disease is frequently reassuring to the clinician, patient, and family. Screening may include a complete blood count and erythrocyte sedimentation rate, stool studies for enteric bacterial pathogens and parasites, and breath hydrogen testing, or a trial of a milk-free diet for lactose malabsorption. During the initial visits, and after establishing rapport with the patient and parents, the clinician inquires about the psychosocial history of the child and family. In patients with intractable symptoms endoscopic evaluation of the colon may be indicated (e.g., colonoscopy and biopsy). If the terminal ileum is not intubated, it may be imaged with small bowel series or white blood cell technetium scan. IBS may occur concurrently in patients with inflammatory bowel disease.

TREATMENT

Once there is a confident diagnosis of IBS, treatment goals are to provide effective reassur- ance and symptom relief. The presence and severity of the pain should be acknowledged. The clinician must educate and reassure the child and family that although IBS causes discomfort, it is not a serious disease. A review of the current understanding of IBS and the exacerbating effects of stress and anxiety on the problem helps the child and family to understand why the pain occurs. ${ }^{27}$ Psychosocial difficulties and triggering events for symptoms should be identified and tackled.

Drug therapy plays an adjunctive role in treatment. Tricyclic antidepressants such as imipramine or amitriptyline in low doses improved symptoms in adults with IBS in controlled, blinded studies. ${ }^{28}{ }^{29}$ These drugs have been used for decades for chronic visceral pain by pain management specialists, but there are only anecdotal reports concerning their use in children with chronic abdominal pain. As amitriptyline has greater sedative and anticholinergic effects than imipramine, amitriptyline may be a better choice for children who wake up at night with pain, or for children with a diarrheal component to their disorder. Imipramine may be a better choice than amitriptyline for children with constipation; at the low doses recommended for chronic visceral pain, chronic constipation is rarely exacerbated. Anticholinergic medications such as dicyclomine, hyoscine, mebeverine, and octylonium have been used for their antispasmodic properties. There are no well designed studies confirming efficacy. In those with constipation, increased dietary fiber (recommended daily fiber intake $=$ age (years) $+5 \mathrm{~g}$ ), milk of magnesia, or mineral oil may be a helpful adjunct.

\section{G2c. Functional abdominal pain}

It should be noted that the term "functional abdominal pain" is not a substitute for Appley's "recurrent abdominal pain of childhood." In some children with functional abdominal pain, the symptoms do not meet the diagnostic criteria for IBS or functional dyspepsia. The pain is usually periumbilical and does not relate to any specific activity; it may keep the patient from sleeping but rarely awakes the patient from sleep. Some of these children are perfectionists, whereas others often have unrecognized learning difficulties; generally, however, the patients or their parents often have high expectations of achievement.

DIAGNOSTIC CRITERIA

At least 12 weeks of:

(1) Continuous or nearly continuous abdominal pain in a school-aged child or adolescent; and

(2) No or only occasional relation of pain with physiological events (e.g., eating, menses, or defecation); and

(3) Some loss of daily functioning; and

(4) The pain is not feigned (e.g., malingering); and

(5) The patient has insufficient criteria for other functional gastrointestinal disorders that would explain the abdominal pain. 
CLINICAL AND DIAGNOSTIC RECOMMENDATIONS The child may also complain of headache, light-headedness, dizziness, nausea (without vomiting), and fatigue. Psychological factors which should be examined include anxiety and/or depression in the child and family, somatization, school phobia, separation anxiety, and secondary gains for illness behavior. Physical examination, growth, and laboratory tests are normal (urinalysis, erythrocyte sedimentation rate, complete blood count, stool examinations for occult blood, leucocytes, ova and parasites, blood chemistries, abdominal ultrasound, and breath hydrogen testing). When symptoms are intractable, further evaluation for organic disease may become necessary, using radiography and endoscopy.

\section{TREATMENT}

Effective reassurance and an explanation for how symptoms occur in the absence of laboratory abnormalities (see earlier) help to establish a therapeutic alliance between the family and the clinician. Psychological support for personal, familial, and school difficulties should be offered when necessary. The best guarantee for success consists of accompanying the children through difficult periods of adjustment and reevaluation whenever symptoms change. The use of a diary to record symptoms, thoughts, and feelings helps the child become involved in his own healing process.

\section{G2d. Abdominal migraine}

Abdominal migraine is a paroxysmal disorder affecting about $2 \%$ of children and characterized by acute, incapacitating, non-colicky, midline abdominal pain that lasts for hours and is accompanied by pallor and anorexia. Associated features include personal and family history of migraine headache. ${ }^{30} 31$

DIAGNOSTIC CRITERIA

(1) In the preceding 12 months, three or more paroxysmal episodes of intense, acute midline abdominal pain lasting two hours to several days, with intervening symptom-free intervals lasting weeks to months; and

(2) Evidence of metabolic, gastrointestinal, and central nervous system structural or biochemical diseases is absent; and

(3) Two of the following features:

(a) Headache during episodes;

(b) Photophobia during episodes;

(c) Family history of migraine;

(d) Headache confined to one side only;

(e) An aura or warning period consisting of either visual disturbances (e.g., blurred or restricted vision), sensory symptoms (e.g., numbness or tingling), or motor abnormalities (e.g., slurred speech, inability to speak, paralysis).

CLINICAL AND DIAGNOSTIC RECOMMENDATIONS When accompanied by a history of migraine headaches, the diagnosis is straightforward; otherwise it should remain presumptive. All other causes of intermittent severe abdominal pain should be considered, including obstructive uropathy, intermittent bowel obstruction, recurrent pancreatitis, biliary tract disease, intracranial space-occupying lesions, familial Mediterranean fever, and metabolic disorders. The diagnosis of abdominal migraine is further supported by a favorable response to medications used prophylactically for migraine.

\section{TREATMENT}

Pizotifen, a serotonin receptor antagonist unavailable in the United States, provides effective prophylaxis. ${ }^{32}$ Cyproheptadine is effective in some patients with migraine headaches and cyclic vomiting syndrome ${ }^{18}$ and might also be helpful in the prophylaxis of abdominal migraine.

\section{G2e. Aerophagia}

Aerophagia consists of excessive air swallowing causing progressive abdominal distension. ${ }^{34}$ Abdominal discomfort is often such that children limit their food intake. This explains why the committee decided to include aerophagia among the abdominal pain disorders.

\section{DIAGNOSTIC CRITERIA}

At least 12 weeks, which need not be consecutive, in the preceding 12 months of two or more of the following signs and symptoms:

(1) Air swallowing; and

(2) Abdominal distension due to intraluminal air; and

(3) Repetitive belching and/or increased flatus.

CLINICAL AND DIAGNOSTIC RECOMMENDATIONS

Aerophagia often goes unnoticed by parents. The physician will observe repeated audible swallows, and inquire about anorexia, abdominal pain, excessive flatus, and excessive burping. The symptoms and abdominal distension resolve during sleep. ${ }^{33}$ Aerophagia may be confused with gastroesophageal reflux disease (because of noises in the throat), and with generalized motility disorders such as chronic intestinal pseudo-obstruction or Hirschsprung's disease (because of associated abdominal distension). A normal physical examination and growth history help exclude disease. Breath hydrogen testing may be useful. It is important to ask about stressful life events because anxiety is a frequent cause of excessive swallowing.

\section{TREATMENT}

Treatment consists of effective reassurance and explanation of the symptom for the parents and child. Often the clinician can help the child become aware of swallows during the visit. Excessive use of chewing gum or carbonated beverages should be discouraged. Stress and anxiety problems should be promptly addressed.

G3. Functional diarrhea (also called toddler's diarrhea, chronic non-specific diarrhea, irritable colon of childhood) Functional diarrhea is defined by daily painless recurrent passage of three or more large, 
unformed stools, for four or more weeks, with onset in infancy or preschool years. There is no evidence of failure to thrive if the diet has adequate calories. The symptom resolves spontaneously by school age. ${ }^{34}$

DIAGNOSTIC CRITERIA

For more than four weeks, daily painless, recurrent passage of three or more large, unformed stools, in addition to all these characteristics:

(1) Onset of symptoms begins between six and 36 months of age;

(2) Passage of stools occurs during waking hours; and

(3) There is no failure to thrive if caloric intake is adequate.

CLINICAL AND DIAGNOSTIC RECOMMENDATIONS

The clinician queries about causes of chronic diarrhea, including enteric infections, ingestion of laxatives, urinary tract infection, and antibiotics. A diet history assesses overfeeding, excessive fruit juice or sorbitol consumption, excessive carbohydrate ingestion with low fat intake, and food allergens. In the absence of failure to thrive, a malabsorption syndrome is unexpected.

TREATMENT

It is important to avoid restrictive diets which may induce caloric deprivation. ${ }^{34}$ In functional diarrhea, a meal fails to interrupt the migrating motor complex. ${ }^{35}$ Children recover spontaneously, and usually no treatment is necessary. Effective reassurance for parents is important. A daily diet and defecation diary helps to reassure them that specific dietary items are not responsible for the symptom.

\section{G4. Disorders of defecation}

Frequency of defecation in healthy infants and children varies with age. ${ }^{36}$ Complaints related to defecatory problems are responsible for $25 \%$ of outpatient visits to pediatric gastroenterologists. ${ }^{37} 38$

\section{G4a. Infant dyschezia}

Infants with dyschezia strain and scream during prolonged attempts to defecate. This behavior persists for up to 20 minutes, until soft or liquid stools pass. This may be repeated several times a day and occurs during the first few months of life. Symptoms resolve spontaneously in a few weeks.

\section{DIAGNOSTIC CRITERIA}

At least 10 minutes of straining and crying before successful passage of soft stools in an otherwise healthy infant less than six months of age.

CLINICAL AND DIAGNOSTIC RECOMMENDATIONS We speculated that this disorder occurs when neonates fail to coordinate increased intraabdominal pressure with relaxation of the pelvic floor. Thus, the complete history (including diet), physical examination (including rectal examination to exclude anorectal abnormalities), and chart of the infant's growth are normal.

\section{TREATMENT}

Parents are reassured by a systematic physical examination completed in their presence. The disorder will resolve when coordination between increased abdominal pressure and relaxation of the pelvic floor is acquired. To avoid perpetuation of dyschezia, maneuvers of rectal stimulation, which produce artificial and potentially noxious sensory experiences, should be discouraged.

\section{G4b. Functional constipation}

Defecation disorder represents the chief complaint in $3 \%$ of pediatric outpatient visits and $10-25 \%$ of pediatric gastroenterology visits. Few reports distinguish between functional constipation and functional fecal retention, so the frequency of functional constipation is unknown. ${ }^{39}$ Children with cerebral palsy often develop functional constipation.

DIAGNOSTIC CRITERIA

In infants and preschool children, at least two weeks of:

(1) Scybalous, pebble-like, hard stools for a majority of stools; or

(2) Firm stools two or less times/week; and

(3) There is no evidence of structural, endocrine, or metabolic disease.

CLINICAL AND DIAGNOSTIC RECOMMENDATIONS In most cases, a thorough history and physical examination are sufficient to diagnose functional constipation. Delayed passage of meconium in a full term newborn raises the suspicion of Hirschsprung's disease. ${ }^{40}$ In a few healthy breast fed infants there may be weeks between bowel movements but the stools are soft. ${ }^{41}$ The transition from breast to formula feedings is often associated with the appearance of functional constipation. However infants on formula feedings and children who pass stools at intervals greater than a week apart and fail to thrive are likely to have an enteric neuromuscular, anatomic, or metabolic disease. Ingestion of medication causing constipation needs to be excluded.

At physical examination, inspection of the back and spine and verification of deep tendon and cremasteric reflexes help to exclude occult spinal dysraphism. At least one anorectal examination is performed allowing evaluation of anatomy, tone, and masses. Laboratory and radiologic examinations are not warranted in the absence of poor weight gain, persistent abdominal distension, fever, Down syndrome, bilious vomiting, or any abnormal physical findings mentioned above. Functional constipation is important because it may predispose to the development of functional fecal retention.

TREATMENT

In infants, fruit juices which contain fructose and sorbitol such as prune and pear increase 
stool water. Barley, corn syrup, lactulose, and sorbitol can also be used, but mineral oil is not recommended..$^{42}$ When solid food is introduced provision of adequate amounts of liquids and fibers is encouraged (age in years $+5=$ number of grams of dietary fiber per day).

\section{G4c. Functional fecal retention}

Functional fecal retention is the most common cause of constipation and fecal soiling in children. It consists of repetitive attempts to avoid defecation because of fears associated with defecation. ${ }^{43}$ Consequently, a fecal mass accumulates in the rectum.

DIAGNOSTIC CRITERIA

From infancy to 16 years old, a history of at least 12 weeks of:

(1) Passage of large diameter stools at intervals $<2$ times per week; and

(2) Retentive posturing, avoiding defecation by purposefully contracting the pelvic floor. As pelvic floor muscles fatigue, the child uses the gluteal muscles, squeezing the buttocks together.

Accompanying symptoms may include fecal soiling, irritability, abdominal cramps, decreased appetite, and/or early satiety. The accompanying symptoms disappear immediately following passage of a large stool.

CLINICAL AND DIAGNOSTIC RECOMMENDATIONS Physical examination allows assessment of rectal fecal mass, which is judged for height above the pelvic brim and consistency with bimanual palpation on either side of the rectus sheath. In these children, who fear painful defecation, the necessity of a rectal examination remains debatable. A neurological examination excludes occult spinal dysraphism as the cause of retentive or non-retentive fecal soiling. Anorectal manometry, anal sphincter ultrasound, or radiography is unnecessary. ${ }^{44}$ The child's behavior during the interview may assist in making the diagnosis. These children appear as if they are unaware and unconcerned, but in reality they are ashamed and feel isolated.

\section{TREATMENT}

Incontinence occurs when stool seeps around the fecal mass and leaks out. Therefore, the clinician, child, and parent should agree on a plan for evacuating the rectal fecal mass, often using daily oral mineral oil. Many toddlers with functional fecal retention are frightened of any anal manipulation, and find enemas particularly scary. Other experts view the fecal mass as an obstruction, and favor enemas and stimulant laxatives to expedite its expulsion. They argue that early passage of the obstructing mass will give the child immediate relief, and provide the confidence necessary for continuing toilet learning. In toddlers, toilet training will not proceed smoothly until the child's fear of painful defecation resolves. Long term maintenance using stool softeners, including mineral oil, lactulose, and colonic lavage solutions assures a prolonged period of painless defecation until the disorder resolves.

\section{G4d. Functional non-retentive fecal soiling}

Functional non-retentive soiling may be a manifestation of an emotional disturbance in a school-aged child. Soiling episodes may have a relationship to the presence of a specific person (e.g., a parent) or time of day, and may represent impulsive action triggered by unconscious anger.

DIAGNOSTIC CRITERIA

Once a week or more for the preceding 12 weeks, in a child older than four years, a history of:

(1) Defecation into places and at times inappropriate to the social context;

(2) In the absence of structural or inflammatory disease; and

(3) In the absence of signs of fecal retention (listed in section G4c).

CLINICAL AND DIAGNOSTIC RECOMMENDATIONS Most children with non-retentive fecal soiling have daily bowel movements and often have complete stool evacuation in their undergarments. There are few complaints of associated constipation and no fecal mass is found upon physical or $x$ ray examination.

\section{TREATMENT}

The goal is to help parents acknowledge the absence of organic disease and accept a referral to a mental health professional for the treatment of emotional problems.

1 Drossman DA, Thompson WG, Talley NJ, et al. Identification of subgroups of functional bowel disorders. Gastroenterol Int 1990;3:159-72.

2 Drossman DA. Creed FH, Fava GA, et al. The functional gastrointestinal disorders: Diagnosis, pathophysiology and treatgastrointestinal disorders: Diagnosis, pathophysiolo
ment. McLean, VA: Degnon Associates, 1994.

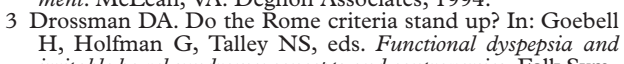
irritable bowel syndrome: concepts and controversies. Falk Symposium 98. Dordrecht: Kluwer Academic Publishers, 1998:11-18

4 Boyce WT, Barr RG, Zeltzer LK. Temperament and the psychobiology of stress. Pediatrics 1992;90:483-6.

5 Liv D, Dioro J, Tannenbaum B, et al. Maternal care hippocampal glucocorticoid receptors and hypothalamicpituitary-adrenal responses to stress. Science 1997;277: 1659-62.

6 Schor E, Starfield B, Stidley C, et al. Family health. Utilization and effects of family membership. Med Care 1987;25: 616-26.

7 Hyman PE, Rasquin-Weber A, Fleisher DR, et al. Childhood functional gastrointestinal disorders. In: Drossman DA, ed. The functional gastrointestinal disorders. McLean, VA: Degnon Associates (in press).

8 Hyman PE (ed). Childhood functional gastrointestinal disorHyman PE (ed). Childhood functional gastrointestinal disor-
ders. New York: Academy Professional Information Servders. New York:
ices (in press).

9 Fleisher DR. Functional vomiting disorders in infancy: innocent vomiting, nervous vomiting, and infant rumination syndrome. F Pediatr 1994;125:S84-94

10 Orenstein SR, Whittington PF. Positioning for preventing infant gastroesophageal reflux. Pediatrics 1982;69:768-72.

11 Orenstein SR, Magill HL, Brooks P. Thickening of infant feedings for therapy of gastroesophageal reflux. $\mathcal{F}$ Pediatr 1987;110:181-6.

12 Scott RB, Ferreira C, Smith L, et al. Cisapride in pediatric gastroesophageal reflux. F Pediatr Gastroenterol Nutr 1997; 25:499-506.

13 Fleisher DR. Infant rumination syndrome. Am $\mathcal{f}$ Dis Child 1979;133:266-9.

14 Sauvage D, Leddet I, Hameury L, et al. Infantile rumination: diagnosis and follow-up study of twenty cases. f Am Acad Child Psychiatry 1985;24:197-203. 
15 Fleisher DR, Matar M. The cyclic vomiting syndrome: A report of 71 cases and a literature review $\mathcal{F}$ Pediatr Gastroenterol Nutr 1993;17:361-9.

16 Pfau BT, Li BUK. Differentiating cyclic from chronic vomiting patterns in children - quantitative criteria and diagnostic implications. Pediatrics 1996;97:367-8.

17 Forbes D, Withers G. Prophylactic therapy in cyclic vomiting syndrome. I Pediatr Gastroenterol Nutr 1995;21(suppl 1):S57-9.

18 Anderson J, Sugerman K, Lockhart JR, et al. Effective prophylactic therapy for cyclic vomiting syndrome in children phylactic therapy for cyclic vomiting syndrome in children using amitr

19 Fleisher DR. Management of cyclic vomiting syndrome. $f$ Pediatr Gastroenterol Nutr 1995;21(suppl 1):S52-6.

20 Carey WB. "Colic" - Primary excessive crying as in infantenvironment interaction. Pediatr Clin North Am 1984;31 993-1005.

21 Appley J, Naish N. Recurrent abdominal pains: A field survey of 1,000 school children. Arch Dis Child 1958;33:16570 .

22 Hyams JS, Burke G, Davis PM, et al. Abdominal pain and irritable bowel syndrome in adolescents: a communitybased study. F Pediatr 1996;129:220-6.

23 Di Lorenzo C, Sigurdsson L, Griffiths J, et al. Rectal and gastric hyperalgesia in children with recurrent abdominal gastric hyperalgesia in children with recurrent

24 Talley NJ, Colin-Jones D, Koch KL, et al. Functional dyspepsia: a classification with guidelines for diagnosis and management. Gastroenterol Int 1991;4:145-60.

25 Graham DY, Malaty HM, Evans DG, et al. Epidemiology of Helicobacter pylori in an asymptomatic population in the United States. Effect of age, race, and socioeconomic status. Gastroenterology 1991;100:1495-501.

26 Sigurdsson L, Flores A, Putnam PE, et al. Postviral gastroparesis: Presentation, treatment, and outcome. $\mathcal{F}$ Pediatr 1997;131:751-3.

27 Walker LS, Garber J, Green JW. Psychosocial correlates of recurrent childhood pain: a comparison of pediatric patients with recurrent abdominal pain, organic illness, and patients with recurrent abdominal pain, organic illness, and 58 .

28 Greenbaum DS, Mayle JE, Vangeren LE, et al. Effects of desipramine on irritable bowel syndrome compared with atropine and placebo. Dig Dis Sci 1987;32:257-66.
29 Pilowsky I, Barroro GG. A controlled study of psychotherapy and amitriptyline used individually in the treatment of chroni

30 Symon DNK, Russell G. Abdominal migraine: A childhood syndrome defined. Cephalagia 1986;6:223-8.

31 Mortimer MJ, Kay J, Jaron A. Clinical epidemiology of childhood abdominal migraine in an urban general practice. Devel Med Child Neurol 1993;35:243-8.

32 Symon DNK, Russell G. Double blind placebo controlled trial of pizotifen syrup in the treatment of abdominal migraine. Arch Dis Child 1995;72:48-50.

33 Gauderer MWL, Halpin TC, Izant RJ. Pathologic childhood aerophagia: a recognizable clinical entity. 7 Pediatr Surg 1981;16:301-5.

34 Lloyd-Still JD. Chronic diarrhea of childhood and the misuse of elimination diets. F Pediatr 1979;95:10-3.

35 Fenton TR, Harries JT, Milla PJ. Disordered small intestinal motility: a rational basis for toddler's diarrhea. Gut motility: a rational

36 Weaver LT. Bowel habit from birth to old age. 7 Pediatr Gastroenterol Nutr 1988;7:637-40.

37 Levine MD. Children with encopresis: A descriptive analysis. Pediatrics 1979;56:412-16.

38 Loening-Baucke V. Chronic constipation in children. Gastroenterology 1993;105:1557-64.

39 Molnar D, Taitz LS, Uurwin OM, et al. Anorectal manometry results in defecation disorders. Arch Dis Child 1983;58: 257-61.

40 Swenson O, Sherman JO, Fisher JH. Diagnosis of congenital megacolon: An analysis of 501 patients. F Pediatr Surg 1973;8:587-94.

41 Hyams JS, Treem WR, Etienne NL, et al. Effect of infant formula on stool characteristics of young infants. Pediatrics 1995;95:50-4.

42 Fan LL, Graham LM. Radiological cases of the month. Lipid pneumonia from mineral oil aspiration. Arch Pediatr Adolesc Med 1994;148:205-6.

43 Partin JC, Hamill SK, Fischel JE, et al. Painful defecation and fecal soiling in children. Pediatrics 1992;89:1007-9.

44 Sutphen J, Borowitz S, Ling W, et al. Anorectal manometric examination in encopretic-constipated children. Dis Colon Rectum 1997;40:1051-2

45 Benninga MA, Buller HA, Heymans HSA, et al. Is encopresis always the result of constipation? Am $\mathcal{F}$ Dis Child 1994; 71:186-93.

\section{For further information and updates on Rome II,}

visit our website at:

www.romecriteria.org 